\title{
Alzheimer's Disease: Errors in Gene Expression
}

\author{
D.R. Crapper McLachlan and P.N. Lewis
}

Can. J. Neurol. Sci. 1985; 12:1-5

Hypotheses concerning the etiology of Alzheimer's disease are numerous but perhaps two of the most probable involve either an unconventional infectious agent of the scrapie, Creutzfeldt-Jakob family or an error in gene expression. Strong circumstantial evidence supports both hypotheses; however, definitive evidence will require an extensive understanding of the molecular biology of this common cause of senile and presenile dementia.

\section{Transmissible Agent Hypothesis}

An unconventional infectious agent is a particularly promising candidate for the primary pathogenic event which initiates the illness because agents of this group do not elicit a systemic immune response, fever, CSF cellular response or nervous tissue inflammatory reaction (Gajdusek, 1977). Furthermore, certain strains of scrapie, in some strains of mice, induce neuritic plaques with amyloid cores similar to those found in Alzheimer's disease (Bruce and Fraser, 1975; Wisniewski et al., 1975). Purification of the infectious moiety indicates that the particle almost certainly does not contain DNA or RNA. The particle is composed of protein which is capable of aggregation into rod-like structures exhibiting the ultrastructural and histochemical characteristics of amyloid (Prusiner et al., 1983). Further, of the six chronic nervous system diseases of mammals known to be associated with amyloid plaques, (Alzheimer's disease, Creutzfeld-Jakob, Kuru, Gerstmann-Straussler syndrome, scrapie and chronic wasting disease in mule deer and elk), only Alzheimer's disease has not been transmitted to other species. Despite the plausible arguments in support of an unconventional transmissible agent, a major difficulty surrounds the argument that neuritic plaques and amyloid formation are central to the etiology of Alzheimer's disease: in each of the transmissible diseases, amyloid formation is an epiphenomenon and the clinical course and disease outcome is independent of the presence or absence of amyloid and neuritic plaque formation. Further, it is not an uncommon histopathological finding that the density of neocortical plaques between elderly non-demented and some severely demented individuals overlap. The correlation between plaque density and behavioral scores in Alzheimer's disease is weak (Roth et al., 1966); one interpretation is that the histological markers may be epiphenomena to the molecular events responsible for the failure in function.

\section{Genetic Hypothesis}

The second hypothesis, an alteration in gene expression, will be examined in some detail. This hypothesis views the primary pathogenic events which initiate the illness to be multifactorial and involves environmental and hereditary factors which operate upon genetically determined regulatory processes resulting in reduced transcription of genetic information. The inevitable consequences of altered gene transcription is the reduction in protein synthesis and loss of cell homeostasis. Such a hypothesis envisages a complex cascade of events leading to cell dysfunction and death.

Fundamental to the regulation of gene expression is the controlled access of the enzyme RNA polymerase to genetic messages encoded within DNA. Access to DNA is controlled by a number of DNA associated proteins which regulate the folding of DNA. Physical states of DNA range from dense compaction of completely repressed genes, through various stages of partial compaction to readily accessible and repeatedly expressed genetic information. Strong circumstantial evidence now indicates that a change in the physical accessibility of DNA to RNA polymerase may explain reduced RNA synthesis and, secondarily, reduced protein synthesis. Reduced protein synthesis may contribute to loss of cell homeostasis through an impairment in the regulation of energy metabolism, intracellular calcium content, neurofilament production and probably other processes which may be important in the pathogenesis of the disease.

\section{Protein Synthesis in Alzheimer's Disease}

Although reduced brain weight, cortical atrophy and enlarged ventricular size are pathological hallmarks of primary degenera- 
tive brain disease, particularly Alzheimer's disease, the most direct evidence for reduced protein synthesis arises from recent studies employing positron emission tomography. This technique has the advantage of measuring in living individuals metabolic processes at various stages of disease, rather than end-stage states that are encountered in studies dependent upon autopsy material. Comar's group at Orsay, France, have developed a dynamic three compartment model of protein synthesis of brain using " $\mathrm{C}$ - $\mathrm{L}$ - methionine as a tracer for human studies (Bustany et al., 1983). Employing 20 control subjects of mean age 80.2 years and 25 demented subjects of mean age 86.3 years, the average incorporation of methionine, expressed as $\mathrm{nM} / \mathrm{min}$ per gram of brain was $\mathbf{0 . 1 5}$ for control and .0 .09 for the demented group. A decrease of more than $65 \%$ in protein synthesis was measured in the most severely demented patients. This study endeavoured to exclude multi-infarct dementia and probably represents predominantly Alzheimer's disease. The frontal-parietal regions exhibited more profound deficits; the occipital lobes were relatively less affected.

While positron emission tomography reveals an overall reduction in protein synthesis, the technique does not indicate whether the deficits involve all groups of proteins or specific subsets of proteins. Furthermore, this technique does not indicate whether a compensatory reduction in protein catabolism accompanies the reduced synthesis. The well documented reduction in the activity of enzymes related to neurotransmission, such as choline acetylase, appears to have resulted from loss of the cells of origin rather than a selective reduction in synthesis or change in activity of the transmitter enzymes (Bowen, 1983). In vitro biochemical analysis of biopsy and autopsy cerebral material from Alzheimer's disease has revealed a marked reduction in enzyme activity related to energy metabolism compared to age matched control tissue (Terry and Davies, 1980). However, studies of soluble cerebral proteins separated by gel electrophoresis or differential centrifugation on sucrose gradients have failed to reveal major qualitative or quantitative changes in Alzheimer affected tissues (Comings, 1982; White et al., 1978; Velde and Stam, 1976; Miner et al., 1976). One interpretation of the biochemical findings to date is that proteins specific to classes of neurons such as neurotransmitter enzymes are selectively reduced but the major soluble structural proteins remain in normal relative proportions. Possibly, cell death has occurred in a cell population which does not significantly differ in relative concentrations of soluble proteins from the surviving population. If so, the observed reduction in the activity of enzymes related to energy metabolism may be a secondary, compensatory, response to the loss of neurons within the tissues affected by the disease and reduced protein synthesis a compensatory change secondary to reduced energy metabolism. An alternative hypothesis considers the primary pathogenic event which initiates the disease to influence protein synthesis. Studies of chromatin structure in Alzheimer's disease and other dementia associated degenerative brain conditions favour this latter possibility.

\section{RNA Content in Alzheimer's Disease}

Measures of both total and messenger (polyA +) RNA in several laboratories indicate a marked reduction in Alzheimer's disease. Oksova (1973) reported a statistically significant $24 \%$ decrease in total RNA content in neurons of layer Ill of neocortex of 10 patients with "senile dementia", compared to 5 age matched control brains. Unfortunately, the histopathology of the brains was not reported. RNA was measured by photometric methods employing gallocyanin stain considered by the author to be specific for RNA phosphate groups and the absorbance measured at $585 \mathrm{~nm}$. Uemura and Hartmann (1979), measured RNA content in neurons dissected from the subiculum of the hippocampus after fixation with formalin. The RNA was extracted from groups of 10 somata with Worthington's ribonuclease and measured with a microdensitometer at 256 $\mathrm{nm}$. These workers reported a mean neuronal RNA content of $73.5 \mathrm{pg}$ in neurons from age matched normal individuals, 41.2 $\mathrm{pg}$ for neurons with neurofibrillary degeneration from brains of individuals with senile dementia of the Alzheimer type and 28.9 $\mathrm{pg}$ for neurons with neurofibrillary degeneration. Mann et al. (1981) measured RNA content in both autopsy and biopsy material from temporal cortex of Alzheimer's disease in formalin fixed tissues employing microphotometric scanning. An average loss of approximately $25 \%$ was noted in layer III and $28 \%$ in layer $\mathrm{V}$.

Direct quantitative measures of RNA from Alzheimer affected tissues has only recently been available. Employing guanidium thiocyanate as RNAase inhibitor, Sajdel-Sulkowska et al. (1983), measured both total RNA and polyA + messenger RNA (mRNA) in control and Alzheimer affected brain. In four young control brains (ages 31-58) and four elderly Alzheimer affected brains (ages 69-90) the recovery of mRNA averaged $2.61 \mathrm{ug} / \mathrm{gm}$ tissue for the Alzheimer material and $10.67 \mathrm{ug} / \mathrm{gm}$ for the control. In this study the control brains were considerably younger than the Alzheimer affected brains and the variable for post mortem RNA autolysis was not reported. In addition to the lower amount of mRNA recovered from Alzheimer tissue, these workers reported that the translational activity in an in vitro reticulocyte protein synthesis system was reduced compared to the control ( $558.449 \mathrm{dpm} / \mathrm{ugRNA}$ for control; $140,000 \mathrm{dpm} / \mathrm{ug}$ RNA for the Alzheimer mRNA). These workers concluded that not only was $m R N A$ recovered in relatively decreased amounts in Alzheimer's disease but under the same in vitro conditions the mRNA is less active than control in the synthesis of protein.

The recovery of RNA and translational competence of mRNA have also been studied in this laboratory. Both the guanidium thiocyanate and the hot phenol methods have been applied. The yield of messenger RNA extracted by the guanidium method from 12 brains of individuals who were either neurologically normal or had a dementia other than Alzheimer's disease and compared to mRNA content extracted from 3 Alzheimer affected individuals revealed a reduction of $51 \%$ in Alzheimer's disease. Employing the phenol method, the mRNA content from the neocortex of 5 control and non-Alzheimer dementia affected brains (mean age $70 \pm 7$ years) was $38 \%$ greater than that extracted from 4 Alzheimer affected brains (mean age $76 \pm 5$ years). This laboratory has not found that the translational competence of Alzheimer mRNA differs from control.

Despite the lability of RNA in postmortem tissues, artifacts associated with freezing of the brain, the agonal process and the several different techniques employed to measure RNA, work in several laboratories supports the conclusion that both total and messenger RNA are reduced in Alzheimer's disease. If RNA were degraded at an accelerated rate in Alzheimer's disease, nucleolar volume would be expected to be increased rather than decreased, as has been regularly observed in Alzheimer affected tissues (Dyan and Ball, 1973; Mann and Sinclair, 1978). 
The weight of evidence favours a reduction in the transcription of RNA in Alzheimer's disease, a conclusion further supported by studies of the structure of chromatin in Alzheimer's disease.

\section{Chromatin Structure in Alzheimer's Disease}

The molecular basis of the control of gene expression in eukaryotes is poorly understood. Genes which are or were being expressed exist in a structural state that is characterized by an increased susceptibility to digestion by nucleases and attack by reactive chemicals (Weisbrod, 1982; Kohwi-Shigematsu et al., 1983). Since the entire human genome is packaged into nucleosomes, it is likely that this increase is due to a greater exposure of the DNA to these reagents as compared to DNA in transcriptionally quiescent chromatin Figure 1 (a). Genes which are being transcribed have a nuclease sensitivity close to that of protein free DNA. The cause of this apparently more open conformation is not known. A number of possible explanations based on the composition of chromatin fractions enriched or depleted in transcriptionally competent genes have been proposed. Active chromatin fractions are characterized by increases in acetylation, ubiquitin, and the high mobility group proteins HMG 14 and 17 (Weisbrod, 1982). As well there are reports of decreased levels of DNA base methylation and the absence of the linker $\mathrm{H} 1$ histones (Ball et al., 1983). Chromatin fractions depleted in transcribed genes are correspondingly enriched in the linker histone $\mathrm{H} l$ group of proteins.

Since it is well known that chromatin depleted in histone $\mathrm{HI}$ will not form the $30 \mathrm{~nm}$ fibre characteristic of condensed chromatin (Bates et al., 1981), it is reasonable to suggest that transcriptionally competent chromatin is less compact. A decrease in compactness is however not the sole determinant of accessibility to a transcribed gene. as it has been shown that the nuclease hypersensitivity can be abolished by salt concentrations well below that needed to remove $\mathrm{H} 1$ from chromatin (Weisbrod and Weintraub, 1981). Thus it is likely that several factors create and maintain the hypersensitive state of transcribing chromatin. For a gene to be transcribed, not only does the gene have to be accessible to RNA polymerase but promoter and enhancer sequences and their specific binding proteins upstream from the gene must also be involved (Emerson and Felsenfeld, 1984). The mechanism whereby a particular gene is identified for transcription is simply not known. It is conceivable that factors such as alternate DNA structures (Nordheim et al., 1981) and the nuclear matrix (Hancock, 1982) are involved in this process. It could be hypothesized that if a histone $\mathrm{H} 1 \mathrm{type}$ protein is bound to a certain region of chromatin, then that region is likely to be transcriptionally inactive.

In Alzheimer's disease two independent techniques have shown that an altered chromatin structure exists in neurons and glia from afflicted cerebral cortex. The first technique (Crapper et al.. 1979) involves the shearing of nuclei and demonstrated
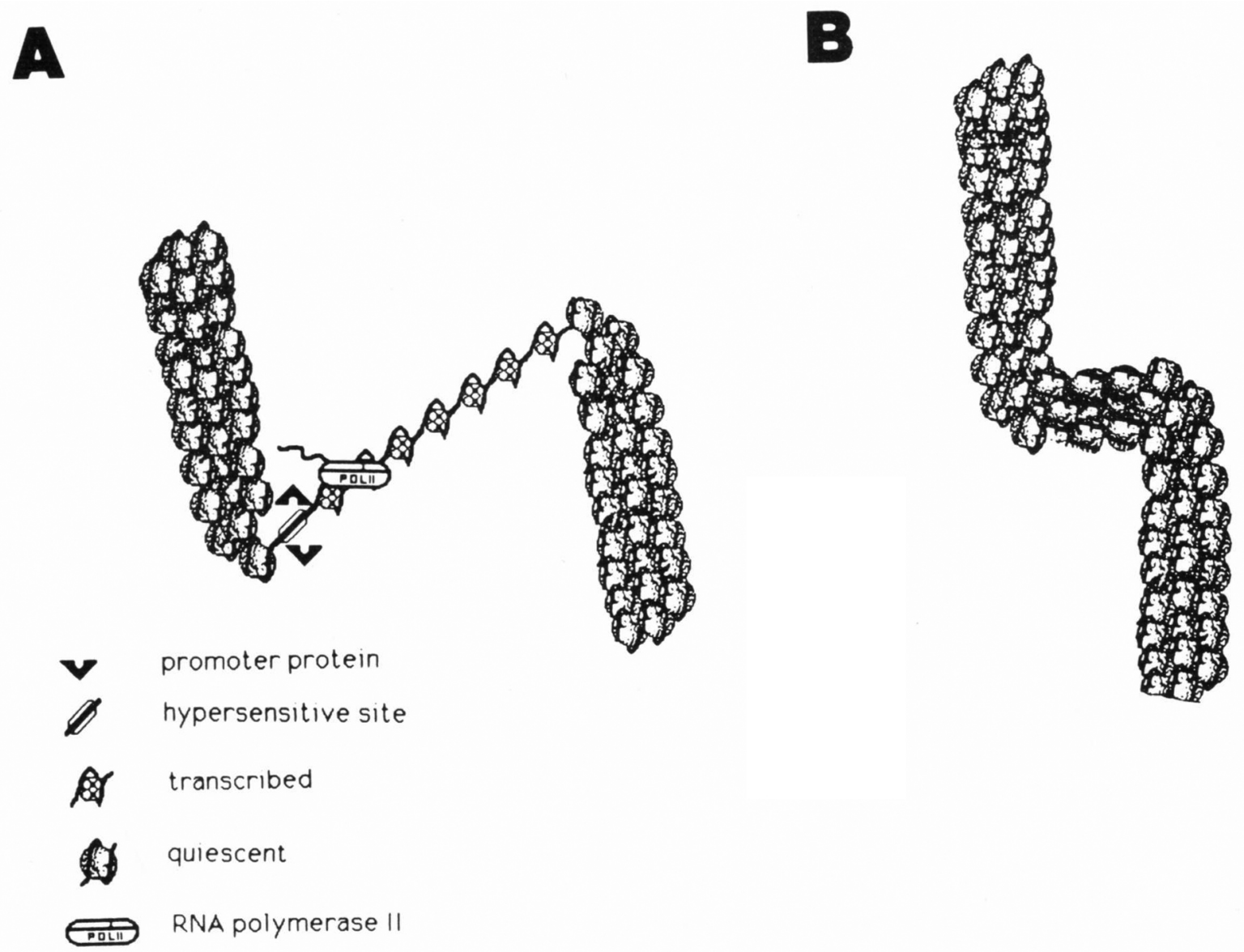

Figure $1-A)$ A transcribing gene flanked by quiescent solenoid chromatin. B) Postulated change in Alzheimer's disease from a normally transcribed gene to an inactive gene associated with histones $H I$, and $H I^{\circ}$. 
that the relative proportions of eu- and heterochromatin from Alzheimer's brain isolated by differential centrifugation differed significantly from that obtained from control brain samples. While about $75 \%$ of the DNA was found in the extended euchromatin from control brains, this percentage decreased to $53 \%$ in Alzheimer brains. This apparent increase in the fraction of compact chromatin was observed for both glia and neurons from Alzheimer afflicted brains.

An enzymatic digestion of chromatin was used as the second technique (Lewis et al., 1981) to distinguish between domains which differ in their susceptibility to digestion and perhaps accessibility to other proteins. The enzyme used in this technique is micrococcal nuclease (EC 3.1.4.7), which preferentially digests the internucleosmal linker DNA. The results of these experiments showed altered kinetics of nuclei digestion for Alzheimer samples. It was found that unlike control nuclei, Alzheimer nuclei displayed a resistance to nuclease digestion which resulted in the accumulation of polynucleosomes, Figure IB. A useful parameter was found to be the ratio of dinucleosomes to mononucleosomes in the digest extract. Alzheimer neocortex nuclei invariably had a ratio of excess of 1 at $10 \%$ digestion while control nuclei have a ratio of about 0.4 . Both glia and neuron Alzheimer nuclei behaved similarly. The dinucleosomes from these digests were isolated and examined for their protein content. It was found that the nuclease resistant dinucleosomes isolated from nuclease digests of Alzheimer nuclei had elevated levels of a methionine containing histone $\mathrm{H} l$ variant called $\mathrm{H}^{\circ}$. This protein has been shown by others to be associated with terminally differentiated cells (Pherson and Cole, 1980), also there are indications that it accumulates with increasing age (Medvedevet al., 1978) and in vitro, at least, $\mathrm{H} 5 \mathrm{a} \mathrm{H}{ }^{0}$ homology condenses chromatin more effectively than the other linker histones (Thomas and Rees, 1983). In addition to Alzheimer's disease, five other degenerative brain diseases that clinically resemble this disease have been similarly examined. The nuclei from brains afflicted by these other diseases do not display alterations in the nuclease digestion (McLachlan et al., 1984) and thus changes in chromatin structure as reflected by the kinetics of nuclease digestion appear to be a marker for Alzheimer's disease.

While nuclei from Alzheimer brains show differences in their nuclease digestion characteristics, there is no apparent change in the total amount of histone $\mathrm{HI}^{\circ}$ present in diseased and control nuclei. Thus one must propose either that the $\mathrm{HI}^{\circ}$ has become redistributed in the Alzheimer chromatin or that other factors are responsible for the altered digestion properties and the presence of histone $\mathrm{HI}^{0}$ is just coincidental. Whatever the explanation, the apparent alteration in the accessibility of a portion of the Alzheimer chromatin suggests the possibility that there might be concommittant changes in gene expression.

To test this possibility, complementary ${ }^{32} \mathrm{P}$ DNA was prepared from messenger RNA extracted from age matched control brain and hybridized to the DNA extracted from control and Alzheimer dinucleosomes. We observed a $180 \%$ increase in hybridization in the Alzheimer sample compared to the control (Guillmette et al., in preparation). This result supports the idea that the nuclease resistant fraction found in micrococcal nuclease digests of Alzheimer nuclei contain genes which are expressed in control brain nuclei. Taken together with the earlier heterochromatization results it seems that in Alzheimer affected brain cells there is a change in the structure of a portion of the chromatin and that genes which are expressed in control cells are associated with the nuclease resistant fraction. Such changes might be the origin of the reduced RNA and protein synthesis mentioned earlier.

Whether the primary event which initiates Alzheimer's disease operates directly on the mechanisms which regulate chromatin structure or operate remotely and the increased condensation is merely one of a group of naturally occurring regulatory mechanisms for controlling transcription, must await further study. Perhaps one of the next most important questions to consider is what genes are affected by inclusion in the Alzheimer nuclease resistant fraction and how do they affect neuronal function.

\section{Virus Versus Genetic Hypothesis: Are They Mutally Exclusive?}

Scrapie is a hydrophobic protein and appears to contain neither RNA nor DNA. This raises the possibility that the agent may be a protein which regulates its own transcription, perhaps through a direct interaction with DNA. The cI and cro proteins of the genetic "switch" known to occur in the bacteriophage lambda might serve as a model for such a mode of action.

In scrapie affected mouse brain there are changes in chromatin structure which are progressive as the disease progresses and resemble those found in Alzheimer's disease. The kinetics of digestion are similar and the profile of dinucleosome linker proteins are also similar in the two conditions. Interestingly, aging in the mouse brain is associated with an increase in the methionine containing histone $\mathrm{H}^{0}$. Employing the $\mathrm{H}^{\circ}$ content on dinucleosomes as the criteria for aging in the mouse brain, scrapie appears to induce "premature aging" in the species.

\section{CONClusion}

Primary degenerative brain disease has remained refractory to human understanding. However, the recent advances in molecular biology have provided powerful new tools for the investigation of these devastating diseases. While the search for the primary pathogenic events which initiate the disease is important, discovery of the cause is unlikely to yield useful therapeutic advances in the near future. Whether the cause turns out to be an inborn error in metabolism expressed late in life or a slow virus, present technology is insufficient to accomplish either successful genetic manipulation or control of an unconventional virus. Perhaps in the short term the contribution of molecular biology will be the understanding of the pathogenesis of the disease for it is in interfering with this aspect of illness that medicine has been traditionally most successful.

\section{ACKNOWLEDGEMENTS}

Supported by the Ontario Mental Health Foundation, Medical Research Council of Canada. Canadian Geriatric Society and the Scottish Rite Charitable Foundation. 


\section{REFERENCES}

Ball DJ, Gross D, Garrard WT (1983) 5-Methylcytosine is localized in nucleosomes that contain histone HI Proc. Natl. Acad. Sci. USA 80: $5490-5494$.

Bates DL, Butler JG, Pearson EC, Thomas JO (1981) Stability of high-order structure of chicken-erythrocyte chromatin in solution. Eur. J. Biochem. 119: 469-476.

Bowen DM (1983) Biochemical assessment of neurotransmitter and metabolic dysfunction and cerebral atrophy in Alzheimer's disease. In Aspects of Alzheimer's Disease. Ed. by R. Katzman. Cold Spring Harbour Laboratory, pp. 219-230.

Bruce MA. Fraser H (1975) Amyloid plaques in the brains of mice infected with scrapie: Morphological variation and staining properties. Neuropath. Applied Neurobiol. 1: 189-202.

Bustany P, Henry JF, Sargent T, Zorifian E. Cabanis E. Collard P. Comar D (1983) Local brain protein metabolism in dementia and schizophrenia: In viro studies with $1 / \mathrm{C}$ - L-methionine and positron emission tomography. In Positron Emission Tomography of the Brain. Ed. by W.D. Heiss and M.E. Phelps. Springer - Verlag. Berlin, Heidelberg, New York, pp. 208-211.

Comings DE (1982) Two dimensional gel electrophoresis of human brain proteins I to V. Clin. Chem. 28: 782-819.

Crapper DR, Quittkat S, De Boni U (1979) Altered chromatin conformation in Alzheimer's disease. Brain 102: 483-495.

Dyan AD, Ball MJ (1973) Histometric observations on the metabolism of tangle bearing neurons. J. Neurol. Sci. 9: 433-436.

Emerson BM, Felsenfeld G (1984) Specific factor conferring nuclease hypersensitivity at the $5^{\prime}$ end of the chicken adult globin gene. Proc. Natl. Acad. Sci. USA 81: 95-99.

Gajdusek DC (1977) Unconventional viruses and the origin and disappearance of Kuru. Science 197: 943-960.

Guillemette JG. Lewis PN, McLachlan Crapper DR. Changes in transcriptional activity associated with the increased heterochromatization found in the neocortex of Alzheimer affected brains. In preparation.

Hancock R (1982) Topological organization of interphase DNA: The nuclear matrix and other skeletal structures. Biol. Cell 46: 105-122.

Kohwi-Shigematsu T, Gelinas R, Weintraub H (1983) Detection of an altered-DNA conformation at specific sites in chromatin and supercoiled DNA. Proc. Natl. Acad. Sci. USA 80: 4389-4393.

Lewis PN, Lukiw WJ. De Boni U. McLachlan Crapper DR (1981) Changes in chromatin structure associated with Alzheimer's disease. J. Neurochem. 37: 1193-1202.

Mann DMA. Sinclair KGA (1978) The quantitiative assessment of lipofusin pigment, cytoplasmic RNA and nucleolar volume in senile dementia. Neuropathol. Appl. Neurobiol. 4: 129-135.

Mann DM. Neary D, Yates PO, Lincoln J, Snowden JS. Stanworth P (1981) Alternations in protein synthetic capability of nerve cells in
Alzheimer's disease. J. Neurology Neurosurgery and Psychiatry 44: $97-102$.

McLachlan Crapper DR, Lewis PN, Lukiw JW, Sima A. Bergeron C. De Boni U (1984) Chromatin structure in dementia. Ann. Neurol. 15: 329-334.

Medvedev ZA. Medvedeva MN, Robson L (1978) Tissue specificity and age changes of the pattern of the $\mathrm{Hl}$ group of histones in chromatin from mouse tissues. Gerontology 24: 286-292.

Miner GD, Trapp GA. McSwigan J. Heston L (1976) Alzheimer's Disease: human brain protein 13-1. J. Neurochem. 26: 605-607.

Nordheim A. Pardue M. Lafer E, Moller A. Stollar B, Rich A (1981) Antibodies to left-handed Z-DNA bind to interband regions of drosophila polytense chromosomes. Nature 294: 417-422.

Oksova EE (1973) Nucleic acid content in cortical neurons in senile dementia. Zhurnal Neuropalotogii i psikhiatrii imeni. S.S. Korsakova, 73(7): 1038-1040.

Pherson JR. Cole RD (1980) Histone $\mathrm{HI}^{\circ}$ accumulates in growth inhibited cultured cells. Nature 205: 43-45.

Prusiner SB. McKinley MP. Bowman KA, Bolton DC, Bendheim PE, Groth DF, Glenner GG (1983) Scrapie prions aggregate to form amyloid-like birefringent rods. Cell. 35: 349-358.

Roth M. Tomlinson BE. Blessed G (1966) Correlation between scores for dementia and counts of senile plaques in central grey matter of elderly subjects. Nature, 5018: 109-110.

Sajdil-Sulkowska EM. Coughlin JF. Staton DM. Marotta A (1983) In Vitro protein synthesis by messenger RNA from Alzheimer's disease brain. In biological aspects of Alzheimer's Disease. Edited by R. Katzman. Cold Spring Harbour Laboratory 193-200.

Terry RD. Davies P (1980) Dementia of the Alzheimer type. Ann. Rev. Neurosci. 3: 77-95.

Thomas $\mathrm{JO}$ and Rees $\mathrm{C}$ ( 1983 ) Exchange of histones $\mathrm{H} \mathrm{I}$ and $\mathrm{H} 5$ between chromatin fragments: A preference of $\mathrm{H} 5$ for higher order structures. Eur. J. Biochem. 134: 109-115.

Uemura E. Hartman HA (1979) Quantitative studies of neuronal RNA on the subiculum of demented old individuals. Brain Research Bulletin 4: 301-305.

Velde OP. Den W. Stam FC (1976) Some cerebral proteins and enzyme systems in Alzheimer's presenile and senile dementia. J. Am. Geriatrics Society $24:$ 12-16.

Weisbrod S (1982) Active chromatin. Nature 297: 289-295.

Weisbrod S. Weintraub H (1981) Isolation of actively transcribed nucleosomes using immobilizated HMG 14 and 17 and an analysis of alpha globin chromatin. Cell 23: 391-400.

Wisniewski HM. Bruce ME, Fraser H (1975) Infections etiology of neuritic (senile) plaques in mice. Science, 190: 1108-1110.

White P. Bowen D, Davison A (1978) Alzheimer's disease: distribution of protein on sucrose density gradient centrifugation. Acta Neuropath. (Berl). 41: 253-256. 